\title{
STUDIES ON THE ISOMERIZATION OF VITAMIN D AND RELATED COMPOUNDS WITH ACIDIC REAGENTS \\ II. MECHANISM OF THE ISOMERIZATION OF VITAMIN $D_{2}$ AND ITS ISOMERS WITH BORON TRIFLUORIDE ${ }^{1}$
}

\author{
Tadashi Kobayashi and Atsuko ADACHI ${ }^{2}$ \\ Department of Hygienic Chemistry, Kobe Women's College of Pharmacy, \\ Higashinada-ku, Kobe 658
}

(Received June 11, 1973)

\begin{abstract}
Kinetic experiments on the isomerization of vitamin $D_{2}$, pre- $D_{2}, 5,6$ trans $-\mathrm{D}_{2}$ and $\mathrm{T}_{2}$ with $\mathrm{BF}_{3}$ were investigated by using the determination method as reported previously (1). The related components in the reaction mixtures were confirmed to be one of the starting compounds, iso- $\mathrm{D}_{2}$ and iso- $\mathrm{T}_{2}$ in all cases by applying the qualitative method using both TLC and GLC. Then, the components were individually determined by the proposed method according to lapse reaction times. The conversion rates of all the starting compounds followed apparent firstorder kinetics and the rate constants of vitamin $\mathrm{D}_{2}$, pre- $\mathrm{D}_{2}, 5,6$-trans $-\mathrm{D}_{2}$ and $T_{2}$ were $0.277,0.462,0.693$ and 0.330 , respectively. When the yields of iso- $\mathrm{D}_{2}$ and iso- $\mathrm{T}_{2}$ to the concentrations of converted starting compounds were calculated on the basis of the corresponding reaction times, the initial rates of yields of iso- $\mathrm{D}_{2}$ and iso- $\mathrm{T}_{2}$ at zero conversions of the starting compounds were respectively 100 and $0 \%$ in all cases. The results induced the conclusions in all cases that iso- $\mathrm{D}_{2}$ was originated as the first reaction product directly from the starting compounds while iso- $\mathrm{T}_{2}$ was the second reaction product originated via iso- $\mathrm{D}_{2}$. The conclusions are summarized as follows:
\end{abstract}

Vitamin $\mathrm{D}_{2}$, pre- $\mathrm{D}_{2}, 5,6$-trans- $\mathrm{D}_{2}$ or $\mathrm{T}_{2} \stackrel{\mathrm{BF}_{3}}{\longrightarrow}$ iso- $\mathrm{D}_{2} \stackrel{\mathrm{BF}_{3}}{\longrightarrow}$ iso- $\mathrm{T}_{2}$

The reaction mechanism was discussed from the conclusions (Fig. 4).

As reported in the previous paper (1), a simple method for the determina-

1 The same abbreviations as described previously (1) are used.

2 小林 正, 足立昌子 
tion of vitamin $\mathrm{D}_{2}$ isomers in mixtures was established. INHOFFEN et al. $(2,3)$ reported that vitamin $D_{2}$, pre- $D_{2}, 5,6$-trans $-D_{2}$, iso- $D_{2}$ and $T_{2}$, which have a 9,10 -seco steroid configuration with a triene system, were isomerized into iso- $\mathrm{T}_{2}$ with $\mathrm{BF}_{3}$ as a typical Lewis acid. On the other hand, we previously reported that the isomerization of vitamin $\mathrm{D}_{2}$ might proceed through the course of vitamin $\mathrm{D}_{2} \rightarrow$ iso- $\mathrm{D}_{2} \rightarrow$ iso- $\mathrm{T}_{2}$ (4). However, the investigation was not quantitatively but qualitatively performed, and therefore the conclusion still remains in an assumption. Moreover, no report has appeared on the mechanism of isomerization of vitamin $\mathrm{D}_{2}$ isomers with $\mathrm{BF}_{3}$. From these considerations, further investigations based on kinetic experiments were performed by using the proposed method. In this paper, the results on the isomerization of vitamin $\mathrm{D}_{2}$, pre- $\mathrm{D}_{2}, 5,6$-trans $-\mathrm{D}_{2}$ and $\mathrm{T}_{2}$ are described.

\section{EXPERIMENTAL}

1. Materials and reagents

$\mathrm{BF}_{3}$-etherate. The commercial grade (guaranteed reagent) of Nakarai Chem. Ind. Co. was used.

Besides it, the same materials and reagents as described previously (1) were used.

2. Procedure for the determination of vitamin $D_{2}$ isomers in mixtures

The same procedure as described previously (1) was used.

\section{RESULTS AND DISCUSSION}

Procedure numbers as described below correspond to those in the previous paper (1).

1. Reaction of vitamin $D_{2}$, pre- $D_{2}, 5,6$-trans- $D_{2}$ and $T_{2}$ with $B F_{3}$

Vitamin $\mathrm{D}_{2}$, pre- $\mathrm{D}_{2}, 5,6$-trans $-\mathrm{D}_{2}$ and $\mathrm{T}_{2}$ chosen as the starting compounds were dissolved separately in benzene to concentrations of $4 \mathrm{mg} / \mathrm{ml}$. The solutions were then made to react with $\mathrm{BF}_{3}$ in the same manner as described in the following:

Ten milliliters of each of the benzene solutions was placed in eight $50 \mathrm{ml}$ volume and glass-stoppered test tubes and then mixed with $10 \mathrm{ml}$ of a $0.2 \% \mathrm{BF}_{3^{-}}$ etherate solution in benzene. After allowing to stand them at a room temperature $\left(19^{\circ} \mathrm{C}\right)$ for $0.25,0.50,0.75,1,5,10,15$ and $20 \mathrm{~min}$, respectively, $10 \mathrm{ml}$ of an $1 \%$ sodium bicarbonate solution was added to each tube to stop the reaction. The separated benzene layers were individually washed several times with small quantities of water and dried over anhydrous sodium sulfate. The benzene solutions thus obtained were denoted as 0.25 to $20 \mathrm{~min}$ reactoin solutions while the solutes in the solutions were denoted as 0.25 to 20 min reaction mixtures, respectively. 


\section{UV spectra of reaction mixtures}

One milliliter of each of the reaction solutions was evaporating to dryness under reduced pressure and then dissolved in $200 \mathrm{ml}$ of ethanol. The UV spectra of the ethanol solutions were then estimated. Their absorption maxima are shown in Table 1, which showed that all the starting compounds were isomerized into iso- $\mathrm{T}_{2}$.

Table 1. Absorption maxima in the UV spectra of reaction mixtures.

\begin{tabular}{lcccc}
\hline \multirow{2}{*}{$\begin{array}{l}\text { Reaction } \\
\text { mixture }\end{array}$} & \multicolumn{4}{c}{ Absorption maxima $(\mathrm{nm})$} \\
\cline { 2 - 5 } & (vitamin $\left.\mathrm{D}_{2}\right)$ & $\begin{array}{c}(\text { Starting } \\
\left(\text { pre- } \mathrm{D}_{2}\right)\end{array}$ & $\left.\begin{array}{c}\text { compound) } \\
(5,6-\text { trans- }\end{array}\right)$ & $\left(\mathrm{T}_{2}\right)$ \\
\hline Starting compound & 265 & 263 & 273 & $270,280,294$ \\
0.25 min & 265 & 263 & 273 & $270,280,294$ \\
0.50 & 265 & 263 & 273 & $270,280,294$ \\
0.75 & $265,287,300$ & 263,287 & $273,287,300$ & $270,280,294$ \\
1 & $265,287,300$ & 263,287 & $273,287,300$ & 280,300 \\
5 & $280,290,302$ & 263,287 & $273,287,300$ & 280,300 \\
10 & $280,290,302$ & 263,290 & $273,287,300$ & $280,290,302$ \\
15 & $280,290,302$ & $280,290,302$ & $280,290,302$ & $280,290,302$ \\
20 & $280,290,302$ & $280,290,302$ & $280,290,302$ & $280,290,302$ \\
\hline
\end{tabular}

3. GLC of reaction mixtures

Two milliliters of each of the reaction solutions were evaporated to dryness under reduced pressure, dissolved in $1 \mathrm{ml}$ of pyridine and the resulting solutions were applied individually to the GLC described in the procedure 3). The peaks observed in each gas chromatogram are shown in Table 2.

\section{TLC and GLC of 5 min reaction mixtures}

In order to confirm the components in the reaction mixtures more definitely, both the TLC and GLC described in procedures 2) and 3) were applied to each of the 5-min reaction mixtures obtained from the starting compounds. Since these reaction mixtures were expected, from the results in Table 2, to contain all the related components in all cases, they were chosen as the representatives of each reaction series. After taking $5 \mathrm{ml}$ of each of the 5-min reaction solutions, evaporating them to dryness under reduced pressure and then dissolving them in $1 \mathrm{ml}$ of $n$-hexane, the resulting solutions were individually applied to the TLC described in the procedure 2). The thin-layer chromatograms are shown in Fig. 1. The zone numbers as shown in the figures correspond to those in the previous paper (1). The parts corresponding to each zone were individually scraped off, treated according to the procedure 2) and then applied to the GLC described in the procedure 3). The gas chromatograms revealed the components from which each zone was originated, as shown in Table 3. The results showed that the components in the reaction mixtures were the starting compounds, iso- $\mathrm{D}_{2}$ and iso- $\mathrm{T}_{2}$ in all cases. These also showed that the procedure without the ap- 
Table 2. Observed peaks in the gas chromatograms of reaction mixtures. Starting compound: vitamin $\mathrm{D}_{2}$

\begin{tabular}{|c|c|c|c|c|c|}
\hline \multirow{2}{*}{$\begin{array}{l}\text { Reaction } \\
\text { mixture }\end{array}$} & \multicolumn{5}{|c|}{ Relative retention time (each peak/S peak) } \\
\hline & $\begin{array}{c}0.28 \\
(\text { pyro-D } \\
2)\end{array}$ & $\begin{array}{c}0.38 \\
\left(5,6-\text { trans }-\mathrm{D}_{2}\right)\end{array}$ & $\begin{array}{c}0.44 \\
\text { (isopyro- } \mathrm{D}_{2} \text { ) }\end{array}$ & $\begin{array}{c}0.51 \\
(\text { iso-- } \\
2 \text { ) }\end{array}$ & $\begin{array}{c}0.59 \\
\left(\mathrm{~T}_{2} \text { or iso- } \mathrm{T}_{2}\right)\end{array}$ \\
\hline Vitamin $\mathbf{D}_{2}$ & + & - & + & - & - \\
\hline $0.25 \mathrm{~min}$ & + & - & + & + & - \\
\hline 0.50 & + & - & + & + & + \\
\hline 0.75 & + & - & + & + & + \\
\hline 1 & + & - & + & + & + \\
\hline 5 & + & - & + & + & + \\
\hline 10 & + & - & + & + & + \\
\hline 15 & + & - & + & - & + \\
\hline 20 & - & - & - & - & + \\
\hline \multicolumn{6}{|c|}{ Starting compound: pre- $_{2}$} \\
\hline Pre-- $D_{2}$ & + & - & + & - & - \\
\hline $0.25 \mathrm{~min}$ & + & - & + & + & - \\
\hline 0.50 & + & - & + & + & + \\
\hline 0.75 & + & - & + & + & + \\
\hline 1 & + & - & + & + & + \\
\hline 5 & + & - & + & + & + \\
\hline 10 & + & - & + & - & + \\
\hline 15 & + & - & + & - & + \\
\hline 20 & - & - & - & - & + \\
\hline \multicolumn{6}{|c|}{ Starting compound: 5,6 -trans $-\mathrm{D}_{2}$} \\
\hline 5,6-trans- $\mathrm{D}_{2}$ & - & + & - & - & - \\
\hline $0.25 \mathrm{~min}$ & - & + & - & + & - \\
\hline 0.50 & - & + & - & + & + \\
\hline 0.75 & - & + & - & + & + \\
\hline 1 & - & + & - & + & + \\
\hline 5 & - & + & - & + & + \\
\hline 10 & - & + & - & + & + \\
\hline 15 & - & - & - & + & + \\
\hline 20 & - & - & - & + & + \\
\hline \multicolumn{6}{|c|}{ Starting compound: $\mathrm{T}_{2}$} \\
\hline $\mathrm{T}_{2}$ & - & - & - & - & + \\
\hline $0.25 \mathrm{~min}$ & - & - & - & + & + \\
\hline 0.50 & - & - & - & + & + \\
\hline 0.75 & - & - & - & + & + \\
\hline 1 & - & - & - & + & + \\
\hline 5 & - & - & - & + & + \\
\hline 10 & - & - & - & - & + \\
\hline 15 & - & - & - & - & + \\
\hline 20 & - & - & - & - & + \\
\hline
\end{tabular}

Note: Compounds described in the parentheses are those corresponding to each peak. 


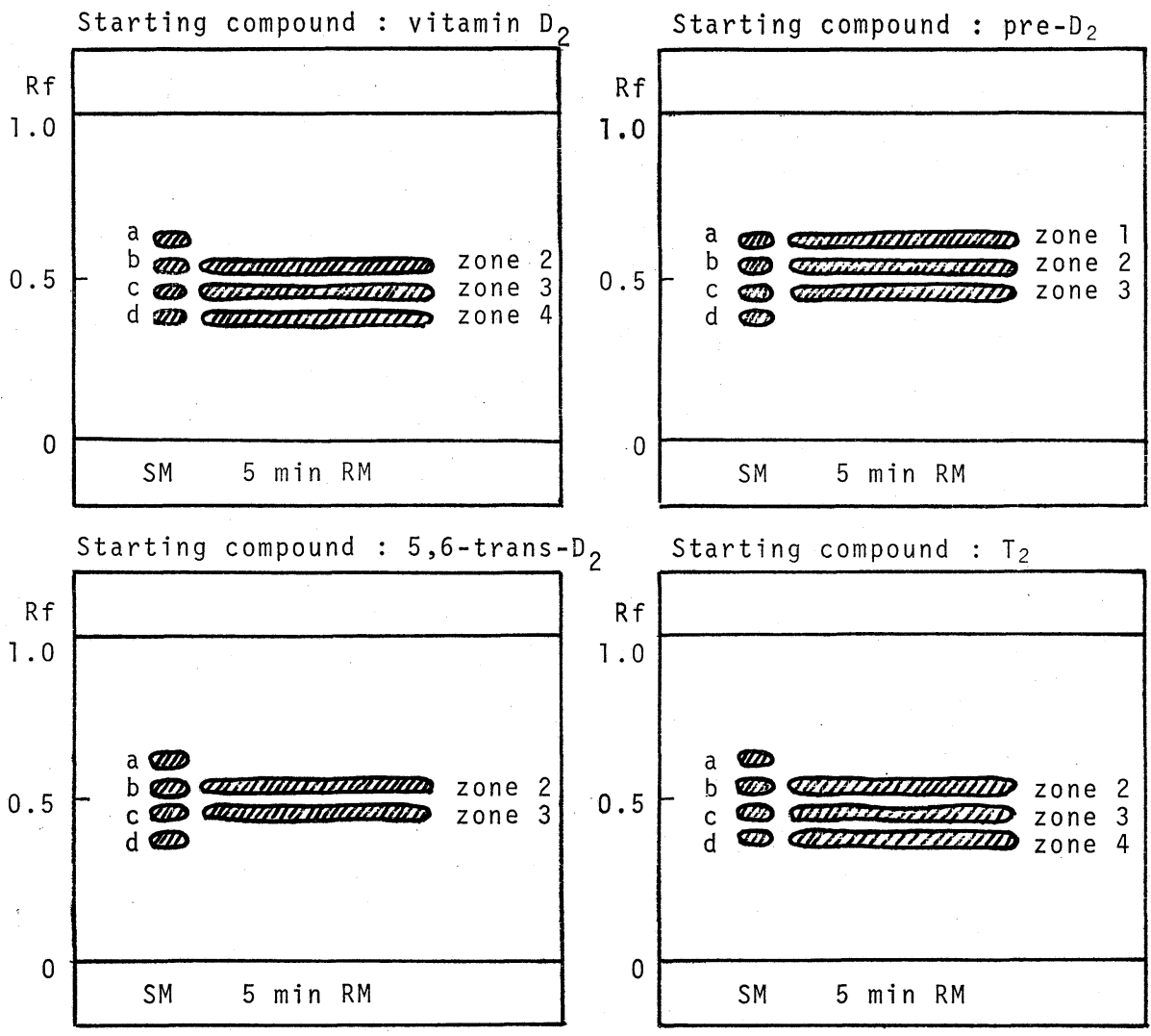

Fig. 1. Thin-layer chromatograms of 5 min reaction mixtures. SM: mixture of standard compounds. a, pre- $\mathrm{D}_{2} ; \mathrm{b}, 5,6$-trans- $\mathrm{D}_{2}+$ iso- $\mathrm{D}_{2} ; \mathrm{c}$, iso- $\mathrm{T}_{2} ; \mathrm{d}$, vitamin $\mathrm{D}_{2}+\mathrm{T}_{2} .5 \mathrm{~min}$ $\mathrm{RM}: 5 \mathrm{~min}$ reaction mixture.

Table 3. Identified compounds in each zone separated by the TLC of $5 \mathrm{~min}$ reaction mixtures.

\begin{tabular}{|c|c|c|c|c|}
\hline \multirow{3}{*}{ Zone number } & \multicolumn{4}{|c|}{ Identified compounds } \\
\hline & \multicolumn{4}{|c|}{ (Starting compound) } \\
\hline & (vitamin $\mathrm{D}_{2}$ ) & $\left(\right.$ pre-- $\left.D_{2}\right)$ & $\left(5,6-\right.$ trans- $\left.\mathrm{D}_{2}\right)$ & $\left(\mathrm{T}_{2}\right)$ \\
\hline Zone 1 & - & pre- $\mathrm{D}_{2}$ & - & - \\
\hline Zone 2 & iso- $\mathrm{D}_{2}$ & iso- $\mathrm{D}_{2}$ & 5,6-trans- $\mathrm{D}_{2}+$ iso- $\mathrm{D}_{2}$ & iso- $\mathrm{D}_{2}$ \\
\hline Zone 3 & iso- $\mathrm{T}_{2}$ & iso- $\mathrm{T}_{2}$ & iso- $\mathrm{T}_{2}$ & iso- $\mathrm{T}_{2}$ \\
\hline Zone 4 & vitamin $\mathrm{D}_{2}$ & - & - & $\mathrm{T}_{2}$ \\
\hline
\end{tabular}

Note: Zone numbers correspond to those described in Fig. 1.

plication of TLC could be used to determine the components in the reaction mixtures obtained from vitamin $\mathrm{D}_{2}$, pre- $\mathrm{D}_{2}$ and 5,6 -trans $-\mathrm{D}_{2}$, because the related components could be separated from one another by the GLC alone. On the other 


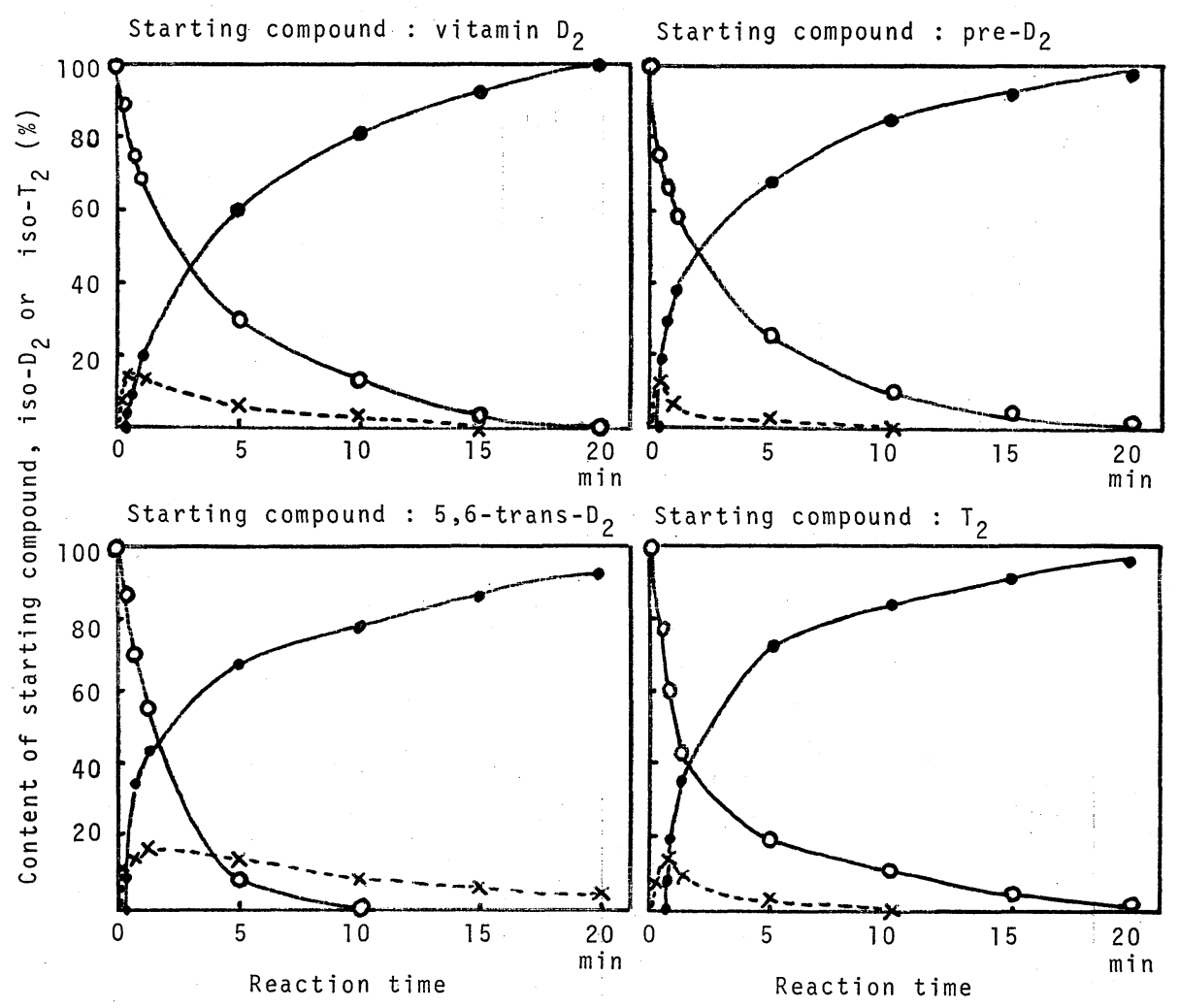

Fig. 2. Contents of starting compound, iso- $\mathrm{D}_{2}$ and iso- $\mathrm{T}_{2}$ in the reaction mixtures.

$\mathrm{O}-\mathrm{O}$, Starting compound; $\times---\times$, iso- $\mathrm{D}_{2}$;

- 1 , iso- $\mathrm{T}_{2}$.

hand, the whole procedure should be applied to determine the components in the reaction mixtures obtained from $T_{2}$, because the separation of $T_{2}$ from iso- $T_{2}$ could not be achieved by the GLC and the two components should be separated from each other previously by the application of TLC.

\section{Determination of each component in the reaction mixtures}

After taking $2 \mathrm{ml}$ of each of the reaction solutions obtained from vitamin $\mathrm{D}_{2}$, pre- $\mathrm{D}_{2}$ and 5,6-trans $-\mathrm{D}_{2}$, evaporating them to dryness under reduced pressure and then dissolving them in $1 \mathrm{ml}$ of pyridine, the resulting solutions were individually applied to the procedure without TLC in order to determine each component in the reaction mixtures. On the other hand, each component in the reaction mixtures obtained from $\mathrm{T}_{2}$ was determined by the application of the whole procedure after taking $10 \mathrm{ml}$ of each of the reaction solutions, evaporating them to dryness under reduced pressure and then dissolving them in $1 \mathrm{ml}$ of $n$-hexane. The results are shown in Fig. 2 in which the percentages calculated as the weight 


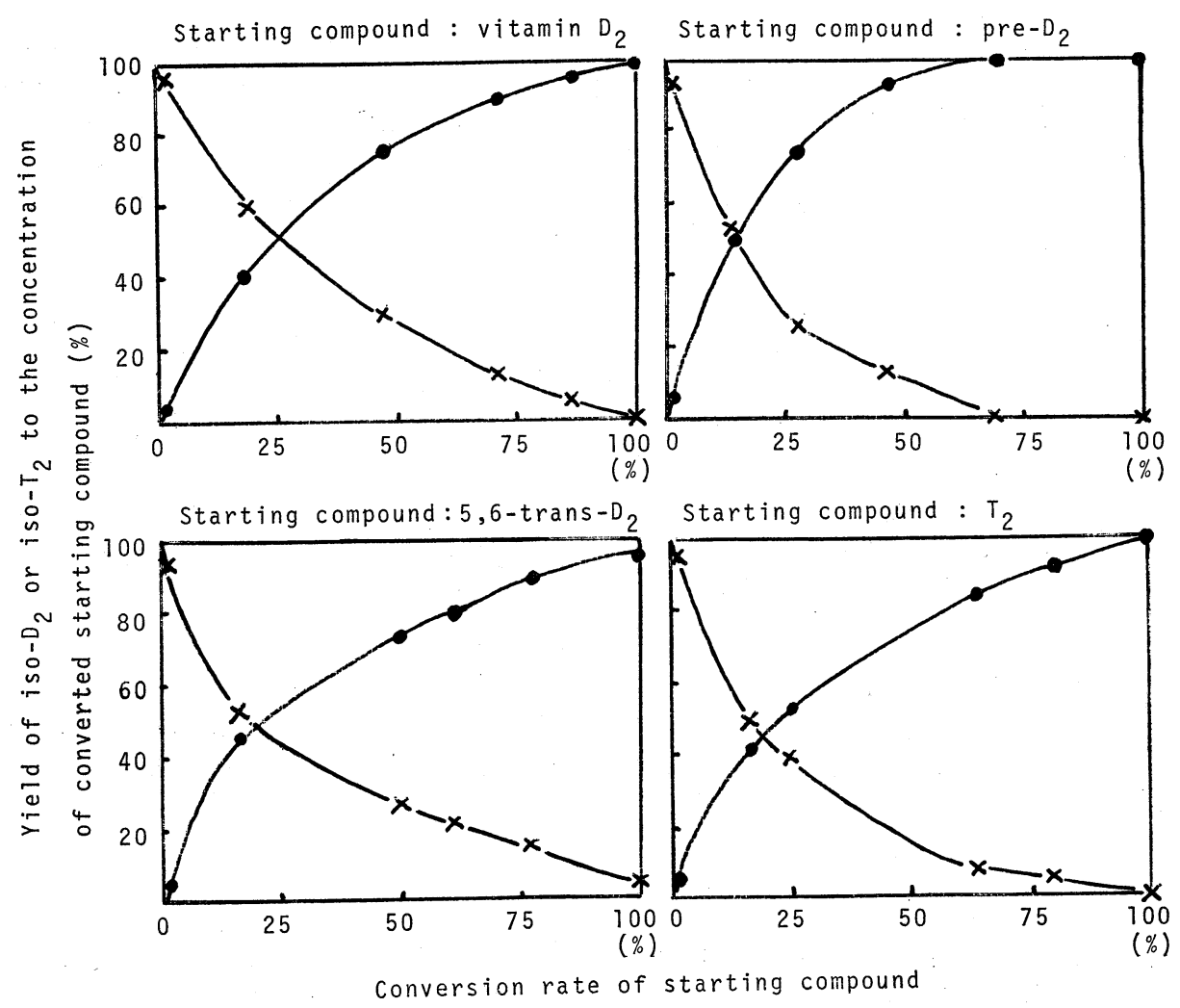

Fig. 3. Yields of iso- $\mathrm{D}_{2}$ and iso- $\mathrm{T}_{2}$ to the conversion rates of starting compound. $x-\times$, iso- $\mathrm{D}_{2} ; \bullet-0$, iso- $\mathrm{T}_{2}$.

Table 4. Rate constant and half-lives for the isomerization of vitamin $\mathrm{D}_{2}$ and its isomers with $\mathrm{BF}_{3}$ at $19^{\circ} \mathrm{C}$.

\begin{tabular}{lcc}
\hline Compound & $k\left(\min ^{-1}\right)$ & $t_{1 / 2}(\min )$ \\
\hline Vitamin $\mathrm{D}_{2}$ & 0.277 & 2.5 \\
Pre- $\mathrm{D}_{2}$ & 0.462 & 1.5 \\
$5,6-$ trans $-\mathrm{D}_{2}$ & 0.693 & 1.0 \\
$\mathrm{~T}_{2}$ & 0.330 & 2.1 \\
\hline
\end{tabular}

ratios of each component to the initial contents of starting compounds are plotted against reaction times. When the relations between the remaining rates of the starting compounds and reaction times were described in a semi-logarithm graph, the conversion rates of all the starting compounds followed apparent first-order kinetics. Their rate constants and half lives are shown in Table 4.

In order to confirm the isomerization courses definitely, the same method as used in the photoisomerization of ergosterol series by RAPPOLDT and HAVINGA 
(5-7) was applied to these cases. According to their method, Fig. 3 was made from the results of Fig. 2 by calculating the yield of iso- $\mathrm{D}_{2}$ or iso- $\mathrm{T}_{2}$ to the concentrations of the converted starting compounds at the corresponding reaction times. Then, if the concentrations of converted starting compound, iso- $\mathrm{D}_{2}$ and iso- $\mathrm{T}_{2}$ at a reaction time are designated as $X, X_{1}$ and $X_{2}$, respectively, Fig. 3 can be made by plotting $X_{1} / X$ or $X_{2} / X \times 100(\%)$ against $X(\%)$.

In Fig. 3, the initial rates of yields of iso- $\mathrm{D}_{2}$ and iso- $\mathrm{T}_{2}$ at zero conversions of the starting compounds were respectively 100 and $0 \%$ in all the four cases. Therefore, the results induced the conclusions in all cases that iso- $\mathrm{D}_{2}$ was originated as the first reaction product directly from the starting compounds while iso- $\mathrm{T}_{2}$ was the second reaction product originated via iso- $\mathrm{D}_{2}$. The conclusions are summarized as follows:

Vitamin $\mathrm{D}_{2}$, pre- $\mathrm{D}_{2}, 5,6$-trans $-\mathrm{D}_{2}$ or $\mathrm{T}_{2} \stackrel{\mathrm{BF}_{3}}{\longrightarrow}$ iso- $\mathrm{D}_{2} \stackrel{\mathrm{BF}_{3}}{\longrightarrow}$ iso- $\mathrm{T}_{2}$

The conclusion in the isomerization of vitamin $D_{2}$ agreed with the assumption as reported previously (5).

From the conclusions, the reaction mechanism on the isomerization of vitamin $\mathrm{D}_{2}$, pre- $\mathrm{D}_{2}, 5,6$-trans $-\mathrm{D}_{2}$ and $\mathrm{T}_{2}$ with $\mathrm{BF}_{3}$ was considered as shown in Fig. 4.

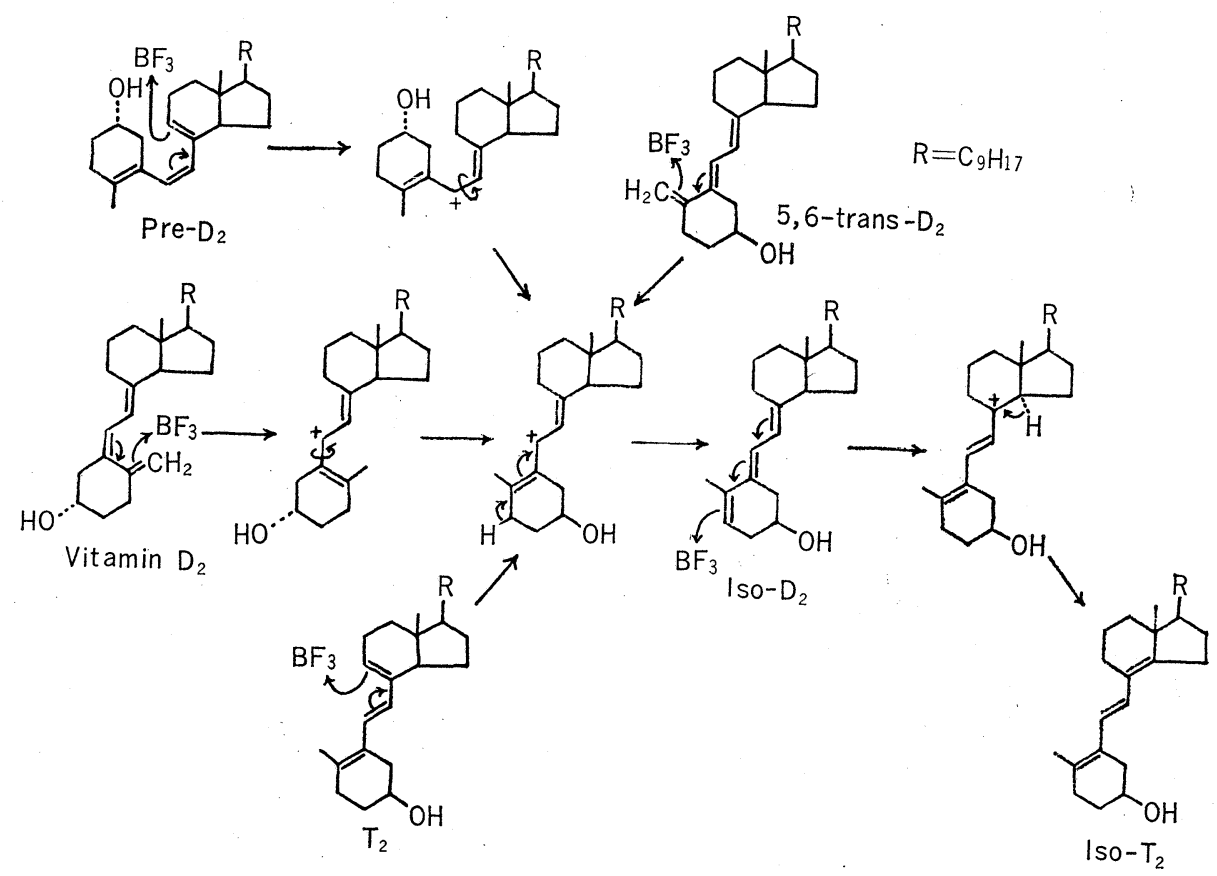

Fig. 4. 
The authors wish to thank Dr. M. Ito of the college for his helpful discussions on the reaction mechanism and Philips-Duphar Co. for gifts of tachysterol 2 -4-methyl-3,5-dinitrobenzoate.

\section{REFERENCES}

1) Kobayashi, T. and Adachi, A., J. Nutr. Sci. Vitaminol., 19, 303 (1973).

2) InHoffen, H. H., BRÜCKNER, K., and GRÜNDEL, R., Chem. Ber., 87, 1 (1954).

3) Inhoffen, H. H., Quinkert, G., Hess, H. J., and Erdmann, H. M., Chem. Ber., 89, 2273 (1956).

4) KobaYASHI, T., Vitamins, 35, 362 (1967).

5) Rappoldt, M.P. and Havinga, E., Rec. Trav. Chim., 79, 369 (1960).

6) Rappoldt, M. P., Rec. Trav. Chim., 79, 392 (1960).

7) Rappoldt, M. P., Rec. Trav. Chim., 79, 1012 (1960). 